\title{
Transparent Photothermal Metasurfaces Amplifying Superhydrophobicity by Absorbing Sunlight
}

\section{Journal Article}

Author(s):

Mitridis, Efstratios (10); Lambley, Henry (1D; Tröber, Sven; Schutzius, Thomas M.; Poulikakos, Dimos

Publication date:

2020-09-22

Permanent link:

https://doi.org/10.3929/ethz-b-000442683

Rights / license:

Creative Commons Attribution 4.0 International

Originally published in:

ACS Nano 14(9), https://doi.org/10.1021/acsnano.0c04365

\section{Funding acknowledgement:}

162565 - The Fundamental Role of Extreme Environmental Conditions on Surface Icing and on the Design of Icephobic Surfaces (SNF)

669908 - Pathways to Intrinsically Icephobic Surfaces (EC) 
This document is the unedited author's version of a submitted work that was subsequently accepted for publication in ACS Nano, copyright (C) American Chemical Society after peer review. To access the final edited and published work, see https://pubs.acs.org/doi/abs/10.1021/acsnano.0c04365 (DOI: 10.1021/acsnano.0c04365).

\section{Transparent Photothermal Metasurfaces Amplifying Superhydro-}

\section{phobicity by Absorbing Sunlight}

Efstratios Mitridis, Henry Lambley, Sven Tröber, Thomas M. Schutzius*, Dimos Poulikakos*

Laboratory of Thermodynamics in Emerging Technologies, Department of Mechanical and Process

Engineering, ETH Zurich, Sonneggstrasse 3, CH-8092 Zurich, Switzerland

* To whom correspondence should be addressed.

Prof. Dimos Poulikakos

ETH Zurich

Laboratory of Thermodynamics in Emerging Technologies

Sonneggstrasse 3, ML J 36

CH-8092 Zürich

SWITZERLAND

Phone: +41446322738

Fax: +41446321176

dpoulikakos@ethz.ch

Prof. Thomas Schutzius

Current address:

ETH Zurich

Laboratory for Multiphase Thermofluidics and Surface Nanoengineering

Sonneggstrasse 3, ML J 27.2

CH-8092 Zürich

SWITZERLAND

Phone: +41 446324604

thomschu@ethz.ch 


\begin{abstract}
Imparting and maintaining surface superhydrophobicity is receiving significant research attention over the last several years, driven by a broad range of important applications and enabled by advancements in materials and surface nanoengineering. Researchers have investigated the effect of temperature on droplet-surface interactions, which poses additional challenges when liquid nucleation manifests itself, due to ensuing condensation into the surface texture that compromises its anti-wetting behavior. Maintaining surface transparency at the same time poses an additional and significant challenge. Often, the solutions proposed are limited by working temperatures or are detrimental to visibility through the surface. Here we introduce a scalable method employing plasmonic photothermal metasurface composites, able to harvest sunlight and naturally heat the surface, sustaining water repellency and transparency under challenging environmental conditions where condensation and fogging would otherwise be strongly promoted. We demonstrate that these surfaces, when illuminated by sunlight, can prevent impalement of impacting water droplets, even when the droplet to surface temperature difference is $50^{\circ} \mathrm{C}$, by suppressing condensate formation within the texture, maintaining transparency. We also show how the same transparent metasurface coating could be combined and work collaboratively with hierarchical micro- and nanorough textures, resulting in simultaneous superior pressure-driven impalement resistance and avoidance of water nucleation and related possible frosting in supercooled conditions. Our work can find a host of applications as a sustainable solution against impacting water on surfaces such as windows, eyewear, and optical components.
\end{abstract}

Keywords: superhydrophobicity, condensation, plasmonic heating, droplet, transparency, sunlight, photothermal 
Inspired by natural examples (lotus leaf, ${ }^{1}$ butterfly wings, ${ }^{2}$ water strider legs ${ }^{3}$ ), superhydrophobic surfaces have received great attention in recent years for their unique self-cleaning, ${ }^{4}$ antifouling, ${ }^{5}$ and anti-icing ${ }^{6}$ properties. Their extreme water repellency, especially with respect to impacting droplets, can be achieved by introducing open and closed microstructures, ${ }^{7-9}$ hierarchical surface roughness, ${ }^{10-14}$ low-surface-energy materials, ${ }^{15-18}$ and substrate flexibility. ${ }^{16,19,20}$ Such surface and substrate properties act to stabilize an intervening, lubricating air layer that is responsible for high droplet mobility (so-called Cassie-Baxter wetting state ${ }^{21}$ ) and prevent a transition to the sticky Wenzel wetting state (i.e. impalement). ${ }^{22}$ For real-world applications, the ability to repel impacting droplets is critical with numerous studies having investigated it under a range of environmental conditions and droplet temperatures ranging from supercooled droplets ${ }^{20}$ and surfaces ${ }^{7,23}$ to ambient ${ }^{24,25}$ and hot droplets. ${ }^{26}$ Typically, impalement is prevented by ensuring that the antiwetting (capillary) pressure is greater than the wetting (droplet) pressure. ${ }^{27}$ However, a host of additional mechanisms responsible for the loss of superhydrophobicity emerge when departing from ambient conditions including condensation within the microtexture when exposed to hot vapor $^{26,28-30}$ and increased viscosity and freezing in the case of supercooled droplets. ${ }^{72,31-33}$ Condensation resistant superhydrophobic surfaces have been demonstrated using nanotexturing through droplet coalescence, ${ }^{34,35}$ scaling the texture to prevent filling within the droplet contact time ${ }^{36,37}$ and minimizing the adhesion force of filled cells. ${ }^{26}$ Yet, all of these approaches fail to prevent the initial nucleation of condensate embryos, limiting their working envelope, especially for high supersaturation conditions. This is a critical issue when imparting nanotexture to a surface, which is routinely done to enhance impalement resistance by increasing the antiwetting capillary pressure. More specifically, when using nanotexture, due to its significantly smaller dimensions compared to microtexture, if a droplet does nucleate within the texture, it fills the roughness very quickly and displaces the lubricating air layer. Consequently, in a supersaturated environment, despite the fact 
that the nanotexture increases the pressure-based impalement resistance, it can actually reduce the repellency of the surface ${ }^{38}$ due to its vulnerability to water nucleation. Moreover, providing multifunctionality by maintaining optical transparency, ${ }^{39,40}$ an important property in many practical applications, is inherently counteractive to imparting superhydrophobicity, since surface coating roughness inherently obscures light by causing scattering. ${ }^{41}$

To address these challenging intertwined problems of condensation-enhanced impalement and the limitations of surface energy and texturing on resisting impalement, while maintaining transparency, we propose an additive approach exploiting unexplored aspects of the involved physics. Inspired by other innovative studies using sunlight for path-breaking applications, ${ }^{42-46}$ includ-

ing catalysis, ${ }^{47}$ desalination, ${ }^{48,49}$ materials synthesis,${ }^{50}$ and ice/fog repellency, ${ }^{51,52}$ we present rationally designed, transparent yet solar light absorbing, superhydrophobic plasmonic metasurface composites. ${ }^{51,52}$ Guided by mass diffusion, heat transfer, and nucleation theories, we investigate the effects of microtexture and nanotexture, substrate subcooling, droplet impact speed, and light intensity on their photothermal operating range and performance. We explore the repellency of water droplets and the mechanisms of texture cavity filling due to condensation across a broad range of temperatures - all while maintaining optical transparency, critical to many functional water repellent surfaces exposed to natural light. We also demonstrate how our coating works in symbiosis with nanotexture to offset inherent undesirable nucleation, which causes failure under moderate supersaturation conditions.

\section{Results and Discussion}

To study the ability of metasurfaces to amplify superhydrophobicity, we fabricated transparent polyurethane acrylate (PUA) micropillared patterns using soft lithography on glass substrates. On top of these, we deposited a well-adhering, ultra-thin metasurface coating with sputter 
deposition. ${ }^{51}$ This coating, relying on surface plasmon effects, significantly enhances broadband light absorption and heating, achieved by nanoengineering of gold nanoparticle inclusions in a titanium dioxide matrix (dielectric); the mean levels of transparency and absorption are controlled by coating thickness. ${ }^{51,52}$ The broadband absorption property is achieved by tuning the size distribution and concentration of the inclusions in titanium dioxide. For concentrations close to the percolation limit (i.e. adjacent nanoparticles forming a continuous electrically conductive path), electronic conductivity increases dramatically, leading to very effective photothermal light absorption. This is important for harvesting the infrared fraction of sunlight, which makes up more than $50 \%$ of its total power, and in contrast to tinted window films and automobile glass, which work byinstead — reflecting infrared. ${ }^{53}$ In a subsequent step, we deposited an ultra-thin conformal low surface energy fluoropolymer coating with initiated chemical vapor deposition (iCVD), resulting in a superhydrophobic plasmonic metasurface composite. Both the metasurface and fluoropolymer coatings do not substantially alter the underlying geometrical features of our micropillared surfaces. (See Methods, Supporting Information, section "Topography of the control”, and Figure S1 for fabrication steps of all types of surfaces used in this study and topography of a superhydrophobic control surface.)

Figure 1a shows a scanning electron micrograph of the plasmonic composite surfaces used in this study. We observe that both the metasurface and fluoropolymer coatings cover the micropillared substrate uniformly, making them almost indistinguishable from the bare pillars. Especially for the metasurface coating, we used low-pressure and high-power sputtering to promote deposition directionality (see Methods for deposition conditions), which ensures coverage of the bottom of the texture, and also to enhance conformality through metal ion resputtering on the sidewalls of the micropillars. ${ }^{54}$ Some submicron features on the surface are due to the soft lithography replication process, but do not negatively impact its performance. (For more information on the 
superhydrophobic control surface, see Supporting Information, section "Topography of the control", and Figure S1.) We measured the apparent static, advancing and receding water contact angle values on the surface to be $\theta^{*}=154^{\circ} \pm 2^{\circ}, \theta_{\mathrm{a}}^{*}=160^{\circ} \pm 2^{\circ}$ and $\theta_{\mathrm{r}}^{*}=141^{\circ} \pm 3^{\circ}$, respectively. For reference, we measured the respective contact angles of a glass substrate coated with a fluoropolymer to be $\theta^{*}=122^{\circ} \pm 2^{\circ}, \theta_{\mathrm{a}}^{*}=125^{\circ} \pm 1^{\circ}$ and $\theta_{\mathrm{r}}^{*}=119^{\circ} \pm 1^{\circ}$. Figure $1 \mathrm{~b}$ shows a schematic of the different layers of our samples: glass substrate, PUA micropillars, metasurface coating, and fluoropolymer coating. The micropillar array has pillars with diameter, $d$, spacing, $s$, and height, $h$ : $[d, s, h]=[2.5,5.0,3.3] \mu \mathrm{m}$. Figure 1c shows a cross-sectional micrograph of a representative metasurface coating with exaggerated thickness (for imaging purposes), revealing gold nanoparticles (bright spots) embedded in a titania matrix. The high optical transparency of our plasmonic composite is evident in the pictures of Figure 1d, where a printed logo was placed directly underneath it, also for the case of a control surface (lacking the metasurface coating), and illuminated from behind.

Figure 1e shows a plot of the transparency, $\mathcal{T}$, reflectivity, $\mathcal{R}$, and absorption, $\mathcal{A}$, vs wavelength (visible and near infrared; 400-2200 nm) for normal light incidence. The mean values of $\mathcal{T}$, $\mathcal{R}$, and $\mathcal{A}$ were $62 \%, 14 \%$ and $24 \%$, respectively, indicating that one can balance transparency with absorption using transparent metasurface coatings ( $\mathcal{T}=51 \%$ in the visible; not opaque). (For more information on the optical properties of the control, see Supporting Information, section "Transparency, reflectivity and absorption of superhydrophobic control surfaces", and Figure S2.) Figure $1 \mathrm{f}$ shows a schematic of the experimental setup used to quantify the effect of illumination on heating of the surface. It consists of a light source (halogen lamp or solar simulator, for low or high power density, respectively; see Methods); an infrared (IR) camera and a pair of thermocouples that measure the sample, $T_{\mathrm{s}}$, and ambient, $T_{\infty}$, temperatures. The normalized irradiance spectra 
of both light sources are shown in the inset. We note that the plasmonic composites absorb at wavelengths between 400 and $2500 \mathrm{~nm}$, which is practically the energy range of the entire solar spectrum. Figure 1g shows plots of $T_{\mathrm{s}}-T_{\infty} v s$ time, $t$, for surfaces illuminated with a solar simulator (power densities: $P=1 \mathrm{~kW} \mathrm{~m}^{-2}$ and $3.5 \mathrm{~kW} \mathrm{~m}^{-2}$; used later in this work). At $t=300 \mathrm{~s}$, we see that, for the plasmonic composite, $T_{\mathrm{s}}-T_{\infty}=4^{\circ} \mathrm{C}$ and $16^{\circ} \mathrm{C}$ for $P=1 \mathrm{~kW} \mathrm{~m}^{-2}$ and $3.5 \mathrm{~kW} \mathrm{~m}^{-2}$, respectively, while, for the same $P$, the $T_{\mathrm{s}}-T_{\infty}$ of control surfaces is at least 3 times lower, demonstrating the significant role of the plasmonically captured sunlight in heating through the photothermal effect. We found that our hydrophobized metasurface adhered very well to substrates after standard tape peel and abrasion tests, and that it still retained its heating property even after partial removal due to scraping (see Supporting Information, section "Mechanical durability of the metasurface coating", and Figure S3). In the following, to avoid unwanted condensation from humidity in the environment, we heat the droplets instead of cooling the substrates to achieve supersaturation. (See also Supporting Information, section "Inhibiting fog with sunlight in plasmonic composite surfaces", and Figure S4.)

Figure 2 a shows an image sequence of a water droplet with an initial radius, $R_{0} \approx 1.6 \mathrm{~mm}$, density, $\rho$, surface tension, $\gamma$, and temperature, $T_{\mathrm{w}}=70^{\circ} \mathrm{C}$, impacting onto our superhydrophobic plasmonic composite with a velocity, $v \approx 0.8 \mathrm{~m} \mathrm{~s}^{-1}$, without illumination $\left(T_{\infty}=21^{\circ} \mathrm{C}\right)$. We define the Weber number, which describes the relative importance of inertia and surface tension during droplet impact, as $W e=2 \rho v^{2} R_{0} / \gamma$. For these experiments, $W e$ was kept constant at 26. The chosen $T_{\mathrm{w}}-T_{\infty}$ means that there is a high supersaturation above the surface and a high probability of condensation under normal circumstances. Figure $2 \mathrm{~b}$ depicts a droplet impacting onto the same surface under identical conditions except that now the surface is illuminated (solar simulator; power density, $P=1 \mathrm{~kW} \mathrm{~m}^{-2}$ ). In Figure $2 \mathrm{a}$, we see that the droplet leaves a remnant on the surface 
(characteristic of impalement), while in Figure $2 b$ the entire droplet rebounds from the surface (see Supporting Information Video S1). Figure 2c plots the probability of droplet impalement on the plasmonic composite, $\Phi$, vs $T_{\mathrm{w}}$, for $P=0$ and $1 \mathrm{~kW} \mathrm{~m}^{-2}$. For $T_{\mathrm{w}}=40^{\circ} \mathrm{C}$, we note the onset of impalement for $P=0 \mathrm{~kW} \mathrm{~m}^{-2}$ while for $P=1 \mathrm{~kW} \mathrm{~m}^{-2}$ we observe no impalement. Therefore, we see that sunlight alone is capable of boosting the existing repellency of a superhydrophobic surface. We attribute impalement to water condensation within the microtexture due to the evaporation of the warmer droplets whose vapor displaces the intervening air layer, as discussed in previous works. ${ }^{26,29}$ (See also Supporting Information, section "Enhanced impalement resistance with concentrated light" and Figure S5 for the effect of concentrated illumination on impalement resistance.)

To further understand and quantify this repellency boosting mechanism, we study next the wetting and impalement behavior of sessile warm droplets as a function of incident light power density, $P$. Figure 3a, Figure 3b, and Figure 3c show image sequences of such droplets with the same initial temperature $\left(T_{\mathrm{w}}=55^{\circ} \mathrm{C}\right)$ for $P=0,1$, and $2 \mathrm{~kW} \mathrm{~m}^{-2}$ (halogen lamp), respectively. The different values of $P, 0,1$, and $2 \mathrm{~kW} \mathrm{~m}^{-2}$, result in different surface temperatures, $T_{\mathrm{s}}$, before droplet placement, of $22^{\circ} \mathrm{C}, 24^{\circ} \mathrm{C}$, and $28^{\circ} \mathrm{C}$, respectively. We define $t_{\mathrm{f}}$ as the duration between placement of a warm droplet onto the surface and the onset of contact line motion. In all previous image sequences, we observe motion for $t=t_{\mathrm{f}}$, accompanied by a decrease in the apparent contact angle and an increased droplet-surface contact area; we presume that this is equivalent to impalement. A clear trend of increasing $t_{\mathrm{f}}$ with increasing $P$ exists. To better investigate this behavior, we can theoretically calculate the approximate timescale needed for condensate, which is generated by the surface being colder than the droplet, to grow and fill the texture. Assuming that this process is diffusion limited, the theoretical filling timescale for constant $T_{\mathrm{s}}$ and $T_{\mathrm{w}}$ can be expressed as ${ }^{26}$ 


$$
\tau_{\mathrm{f}}=\rho h^{2} / D_{\mathrm{v}} \Delta C
$$

where $D_{\mathrm{v}}$ is the water vapor diffusion coefficient in air and $\Delta C$ is the water vapor concentration difference between the droplet and the bottom of the texture. $\Delta C$, in turn, is a function of the local water vapor saturation pressure difference across a cavity, $\Delta p_{\mathrm{v}, \mathrm{sat}}$, which is a function of both the droplet and substrate temperatures. It is noted that $\tau_{\mathrm{f}}$ strongly depends also on the pillar height, scaling as $h^{2}$. (For more details on $\tau_{\mathrm{f}}$, see Supporting Information, section "Theoretical basis of impalement criterion".)

Figure $3 \mathrm{~d}$ shows a plot of $t_{\mathrm{f}} v s \tau_{\mathrm{f}}$ for three different values of $P\left(0,1\right.$, and $\left.2 \mathrm{~kW} \mathrm{~m}^{-2}\right)$. Lines of best fit are shown for these three cases. We varied the values of $\tau_{\mathrm{f}}$ by varying $T_{\mathrm{w}}$; these two quantities have an inverse correlation. Figure $3 \mathrm{~d}$ provides a quantitative relationship between $t_{\mathrm{f}}$ and $\tau_{\mathrm{f}}$, from which we define $\alpha=t_{\mathrm{f}} / \tau_{\mathrm{f}}$ as an empirical correlation coefficient, and their dependence on $P$. In relative agreement with previous research on hot droplets interacting with ambient substrates, ${ }^{26}$ we found $\left.\alpha\right|_{T_{\mathrm{s}}=T_{\infty}=22^{\circ} \mathrm{C}} \approx 11$. However, for $T_{\mathrm{s}}>T_{\infty}$, which occurs naturally due to the surface being illuminated, we observed that $\alpha$ increases significantly to $\left.\alpha\right|_{T_{\mathrm{s}}=28^{\circ} \mathrm{C}} \approx 26$ (see Supporting Information, sections "Effect of surface temperature on condensation nucleation on superhydrophobic control surfaces" and "Experimental setup, water temperature calibration and droplet cooling rate", Figure S6 and Figure S7). Figure 3e shows schematically the mechanism of droplet impalement due to condensation from a warm droplet over time inside the surface texture. For contact times, i.e. the duration of the touch of the droplet on the surface, $t_{\mathrm{c}}<<t_{\mathrm{f}}=\alpha \tau_{\mathrm{f}}$, the cavities are almost water-free and superhydrophobicity is maintained. As $t_{\mathrm{c}}$ and $\alpha \tau_{\mathrm{f}}$ become 
comparable, condensation occurs and the cavity begins to fill with water, while for even longer $t_{\mathrm{c}}$ , water completely fills the cavity and local superhydrophobicity is compromised.

To explore a potential coupling of pressure and condensation-based impalement mechanisms, we mapped the impalement probability, $\Phi$, of warm droplets impacting onto our plasmonic composites for a range of $W e$. The image sequences in Figure $4 \mathrm{a}$ and Figure $4 \mathrm{~b}$ depict droplets with $T_{\mathrm{w}}>T_{\mathrm{s}}$ impacting onto a surface at $W e=73$ for $P=0$ and $3.5 \mathrm{~kW} \mathrm{~m}^{-2}$, respectively. We used a concentrated illumination of 3.5 suns to better demonstrate any possible effects of light on condensation-based impalement. We see that even though the droplet is warmer in the case of the illuminated sample, and therefore more likely to impale, the value of $P$ used here is sufficient to suppress impalement and boost the naturally existing repellency of the surface. To isolate the pressure-based impalement mechanism, we performed droplet impact experiments in isothermal conditions ( $T_{\mathrm{w}}=T_{\mathrm{s}}=T_{\infty}$ ) for $W e=73$, which revealed a significant impalement probability, $\Phi \approx 86 \%$. (See Supporting Information, section "Effect of light on pressure-driven impalement in plasmonic composite surfaces" and Figure S8. See also section "Comparison of droplet retraction dynamics" and Figure S9 for the relationship between droplet recoil dynamics and outcome of an experiment, impalement $v s$ rebound.)

In order now to develop a theoretical predictor of dynamic droplet impact impalement events, either pressure or condensation-based, we additionally define the theoretical droplet oscillation time as $^{20} \tau=(\pi / 4) \sqrt{\rho R_{0}^{3} / \gamma}$, which is a measure of droplet-substrate contact time during impact (see also Supporting Information, section "Theoretical basis of impalement criterion"). A comparison between the droplet contact and cavity filling theoretical timescales, where the latter has been corrected by the previously calculated correlation coefficient $(\alpha), \tau / \alpha \tau_{\mathrm{f}}$, constitutes our predictive impalement criterion. Figure $4 \mathrm{c}$ shows a plot of $\Phi v s \tau / \alpha \tau_{\mathrm{f}}$, for warm droplet impact 
events at $W e=26$ and 73, for light off and light on cases, $P=0$ and 1 or $3.5 \mathrm{~kW} \mathrm{~m}^{-2}$, respectively. When $T_{\mathrm{w}}$ is increased, $\tau / \alpha \tau_{\mathrm{f}}$ also increases. Were the model proposed to capture all the physics involved, impalement transition would be expected to occur for $\tau / \alpha \tau_{\mathrm{f}} \approx 1$, with full impalement ( $\Phi=100 \%$ ) predicted at values $\geq O(1)$. The lines for different experimental conditions should collapse into one. In practice, we observe this behavior in the onset of impalement transition for both light off and light on cases at low $W e$, for $\tau / \alpha \tau_{\mathrm{f}} \in\left[10^{-2}, 1\right)$; the maximum droplet temperature we could repeatably achieve experimentally limits us to values $<1$. For the high $W e$ cases, however, the onset is at $\tau / \alpha \tau_{\mathrm{f}} \approx 10^{-2}<1$, with concentrated illumination mildly decreasing $\Phi$. This behavior can be explained through the schematics in Figure $4 \mathrm{~d}$ and Figure 4e. At low We, the condensationdriven mechanism is dominant and impalement can be fully predicted by $\tau / \alpha \tau_{\mathrm{f}}$. However, at higher $W e$, a high wetting pressure results in meniscus penetration, to a depth, $\delta$, into the texture during impact, effectively reducing the cavity height needed to be filled with water for impalement to occur. This reduces $\tau_{\mathrm{f}}$, which —in this case-is $\sim(h-\delta)^{2}$, resulting in an underestimated $\tau / \alpha \tau_{\mathrm{f}}$. We term this mechanism combined, as there are condensation and pressure components acting simultaneously. It would be possible to use the same impalement criterion for the combined mechanism by replacing $h$ with $h-\delta$. For example, if $\delta=0.8 \cdot h, \tau / \alpha \tau_{\mathrm{f}}$ increases twenty-fivefold; this would shift impalement transition towards unity. Regardless, for the scope of this study, our criterion holds well for order of magnitude analyses involving cavity filling. (See also Supporting Information, section "Mapping droplet impact behavior on plasmonic composite surfaces", and Figure S10 for a comprehensive dynamic droplet repellency performance map of plasmonic composites.) 
We will now explore strategies to fundamentally address the pressure-based impalement mechanism. Figure 5a and Figure 5b show image sequences of ambient droplets with $T_{\mathrm{w}}=T_{\infty}=21^{\circ} \mathrm{C}$ impacting onto our control surface (no metasurface coating) for $W e=26$ and $W e=73$, respectively; the surfaces are not illuminated $\left(P=0 \mathrm{~kW} \mathrm{~m} \mathrm{~m}^{-2}\right)$. We observe droplet rebound in the low $W e$ case, while, in contrast, at $W e=73$, a daughter droplet remains on the surface owing to the pressure driven impalement mechanism. To address this, we improved the superhydrophobicity of the single-tier plasmonic composite by spray coating a dispersion of silanized (hydrophobic) silica nanoparticles with a polymeric binder for enhanced durability. Adding a second tier of roughness does not compromise surface transparency; see inset logo picture in Figure 5c. Figure $5 \mathrm{c}$ and Figure $5 \mathrm{~d}$ depict water droplets of temperature, $T_{\mathrm{w}}=21^{\circ} \mathrm{C}$ and $32^{\circ} \mathrm{C}$, respectively, impacting onto our hierarchical plasmonic composite for $W e=95$, a more vigorous test. We notice the effect of nanoroughness in the increased impalement resistance, evident by the full repellency of the ambient water droplet ( $n=7$ experiments) at high $W e=95$, Figure $5 \mathrm{c}$. However, raising $T_{\mathrm{w}}$ to $32^{\circ} \mathrm{C}$ leads to $100 \%$ impalement probability $(n=7)$, Figure 5 d. Finally, Figure 5 e shows how effectively sunlight ( $\left.P=1 \mathrm{~kW} \mathrm{~m}^{-2}\right)$, for the same $W e$ and $T_{\mathrm{w}}$ as in Figure $5 \mathrm{~d}$, helps mitigate cavity filling and impalement by drastically reducing $\Phi$ to $28 \%$.

In order to explain the previous - unexpected-impalement events and also the effect of illumination, we investigated the nanocavity filling dynamics. From an extended view of the inset nanoroughness micrograph in Figure 5c-e, and assuming for simplicity cylindrical nanopores with an aspect ratio of unity, we measured the average nanopore diameter and subsequently depth to be $\approx 50 \mathrm{~nm}$. To determine the dominant water vapor mass transfer mechanism at these small length scales, we define the Knudsen number, $K n=\lambda_{\mathrm{f}} / d_{\mathrm{r}}$, which compares $d_{\mathrm{r}}$ to the molecular mean free path in saturated moist air, $\lambda_{\mathrm{f}}$. For saturated ambient conditions, ${ }^{55} K n \approx 1.5$, rendering a 
continuum mechanics approach inapplicable, since for $K n>0.5$, the dominant mechanism is free molecular flow. ${ }^{56}$ The correct theory for describing such flows is based on effusion, ${ }^{57}$ and with this the lower bound of the nanopore filling time, $\tau_{\mathrm{fe}}$, is very short $\left(\sim 10^{-4} \mathrm{~s}\right)$. (See Supporting Information, section "Effusion in a nanopore" for the calculation method of the nanopore filling time due to effusion.) For $T_{\mathrm{w}}=32^{\circ} \mathrm{C}$ and $T_{\infty}=21^{\circ} \mathrm{C}$, we calculate $\tau / \tau_{\mathrm{f}, \mathrm{e}} \approx 10^{3} \gg 1$, indicative of impalement, considering effusion alone. For nanopore filling to be initiated, we must verify that condensation nucleation occurs at a significant rate. Based on classical nucleation theory, Figure $5 \mathrm{f}$ plots $P$ vs $T_{\mathrm{w}}-T_{\infty}$ vs $N$, where $N(=\tau A J$, where $J$ is the heterogeneous condensation nucleation rate [nuclei per area per time] and $A$ is the surface area of a single cylindrical nanopore) is defined as the number of water nuclei per nanopore, formed during droplet impact. $T_{\mathrm{w}}$ was kept constant at $32^{\circ} \mathrm{C}$ and $\Delta T=T_{\mathrm{w}}-T_{\infty}$ was varied by changing the temperature of the colder environment, $T_{\infty}<T_{\mathrm{w}}$. (See also Supporting Information, section "Heterogeneous condensation nucleation".) A local static water contact angle, $\theta=120^{\circ}$, corresponding to minimum wetting in the nanoscale, due to the hydrophobic nanoparticles, was considered. We calculated the radius of curvature of the nanopits as $4 \mathrm{~nm}$ using the Brunauer-Emmett-Teller theory for the silica nanoparticle powder and assuming spherical and monodisperse nanoparticles. Furthermore, a linear relationship between $P$ and surface temperature applies. Defining $N=1$ [nuclei per nanopore per contact time] as probable for condensation nucleation based impalement, the non-illuminated surface can only sustain a non-wetting state until $\Delta T \approx 8^{\circ} \mathrm{C}$, which explains the impalement events we observed (Figure $5 \mathrm{~d}$ ). For this $\Delta T$, nanopore filling is nucleation limited - in contrast to the single-tier geometry. Solar illumination of one sun boosts $\Delta T$ by $4^{\circ} \mathrm{C}$ and prevents nucleation in the first place, which, in turn, prevents impalement (Figure 5e). 
The advantages of our plasmonic composite coating extend beyond ambient conditions into supercooled environments, where freezing is also probable to occur if condensation is not controlled. For $T_{\mathrm{w}}=0^{\circ} \mathrm{C}$, we compute a minimum working $T_{\infty}$ of $-7^{\circ} \mathrm{C}$ without and $-11^{\circ} \mathrm{C}$ with solar illumination. This $4^{\circ} \mathrm{C}$ shift is very important, considering the exponential dependence of condensation nucleation rate, $J$, on surface temperature. In fact, heating by just $1^{\circ} \mathrm{C}$ can reduce nucleation rates by up to several orders of magnitude for both condensation and freezing processes, ${ }^{51,52,58}$ emphasizing how sunlight can be effectively and passively employed to prevent impalement on surfaces that typically forgo condensation resilience in pursuit of superior pressure-based impalement resistance.

\section{Conclusions}

We demonstrated a facile and passive method for achieving superior water repellency while maintaining surface transparency in supersaturated environments, relying on the collaborative effect of superhydrophobicity and passive heating through plasmonic exploitation of the photothermal effect stimulated by sunlight. Harvesting solar power with ultrathin plasmonic metasurface coatings without being detrimental to transparency, we demonstrated that typical microstructures can sustain superhydrophobicity and avoid droplet impalement at substrate temperatures much lower than the droplet temperature by fully preventing or retarding cavity filling in the diffusionlimited regime due to light absorption heating. We then discovered that adding a second tier of roughness to our micropillars, intended to boost the superhydrophobicity of the surface against high Weber number (impact velocity) incoming warm droplets, counterintuitively caused the surface to be less repellent by shifting cavity filling to a nucleation-limited regime. We established that by using a metasurface coating, one could inhibit condensation nucleation and significantly expand the working envelope of such hierarchical superhydrophobic surfaces, especially for 
supercooled environments, where it would have the added benefit of reducing the likelihood of freezing. Our approach can be used as a stand-alone or additive method towards counteracting the negative effects of warm(er) water vapor condensation on cold(er) surfaces and whenever a good degree of optical transparency is required. We believe that our work can make its advantages evident in a plethora of applications including glasses, optical components, windows, and windshields exposed to warmer humid conditions.

\section{Methods}

Materials

Microscope glass slides (76 by $26 \mathrm{~mm}, 1-\mathrm{mm}$ thick, with cut edges and plain end) were obtained from VWR. Polydimethylsiloxane (PDMS) silicone elastomer and curing agent (Sylgard ${ }^{\circledR}$ 184, 10 to 1 ratio) were purchased from The Dow Chemical Company, and a UV-curable polyurethane acrylate (PUA) resin (MINS-311RM), composed of a functionalized pre-polymer, a photoinitiator and a UV-curable releasing agent, was obtained from Minuta Technology. Trichlorovinylsilane (TCVS, 97\%), 1H,1H,2H,2H-perfluorodecyl acrylate (PFDA, 97\%, with tert-butylcatechol inhibitor), tert-butyl peroxide (TBPO, 98\%) and ethanol (96\%, anhydrous) were sourced from Sigma-Aldrich. Hydrophobic fumed silica (HFS) nanoparticles (Aerosil ${ }^{\circledR}$ R 812) were purchased from Evonik Industries and Capstone ${ }^{\circledR}$ ST-100HS aqueous fluoropolymer dispersion from DuPont.

\section{Surface preparation}

a. Plasmonic composite surfaces. We fabricated a specially designed chrome hard mask consisting of 20 by $20 \mathrm{~mm}$ squares filled with circular dots with a target diameter of $2.5 \mu \mathrm{m}$ and spacing ranging from $\approx 5 \mu \mathrm{m}$ to $\approx 10 \mu \mathrm{m}$ and used it to pattern 4-in silicon wafers with standard 
photolithography. We used a positive photoresist (AZ 4512; MicroChemicals GmbH) and the Süss MA6 mask aligner in hard contact mode for the UV exposure, and subsequently etched the wafers (deep silicon etch; PlasmaPro 100 Estrelas, Oxford Instruments) to a depth of $3.2 \pm 0.1 \mu \mathrm{m}$. We then used initiated chemical vapor deposition (iCVD; iLab Coating System, GVD Corporation) to coat them with a thin and ultra-low surface energy pPFDA $\left(9.3 \mathrm{mN} \mathrm{m}^{-1}\right){ }^{59}$ conformal hydrophobic polymer layer. Upon a 10-min oxygen plasma ashing (100 W power; PCCE, Diener) of a wafer, we placed it in a glass chamber and ran a room temperature TCVS pre-treatment silanization process with initial pressure of $\sim 13 \mathrm{kPa}$ for $20 \mathrm{~min}$, which would later enable chemical bonding of the pPFDA to silicon. For the iCVD deposition, the reactants were PFDA (monomer) and TBPO (initiator), and the parameters were the following: deposition time of $15 \mathrm{~min}$, process pressure of $\sim 13$ $\mathrm{kPa}, \mathrm{PFDA}$ flow rate of $1.0 \mathrm{sccm}$, TBPO flow rate of $2.6 \mathrm{sccm}$, and substrate temperature of $40^{\circ} \mathrm{C}$. A heated filament at $300^{\circ} \mathrm{C}$ enabled breakdown of the reactants. The next step consisted of transferring the wafer pattern onto a flexible PDMS mold by means of soft lithography, for the preparation of which we mixed PDMS with a curing agent at a 10:1 ratio, poured it onto the silicon wafer and degassed under vacuum to remove all air bubbles. Curing was done in a convection oven at $70^{\circ} \mathrm{C}$ for $2 \mathrm{~h}$. The mold, consisting of microholes, was peeled off the wafer. We then transferred the pattern from the flexible mold onto our substrates. We took microscope glass slides and thoroughly cleaned them, consecutively, in sonicated acetone, isopropyl alcohol and water baths, followed by plasma ashing for $10 \mathrm{~min}$. Subsequently, we placed fractions of the PDMS mold onto thin layers of PUA resin, which we previously deposited on the glass slides. Curing and thus hardening of the pattern (micropillars) took place in a vacuum UV exposure box (Gie-Tec $\mathrm{GmbH}$ ) for 10 min, followed by peeling off the PDMS molds. In order to deposit our light-absorbing metasurface coating on top of our structures, we employed sputter deposition (CS 320 C; Von Ardenne) method. ${ }^{51}$ We first deposited $\sim 20 \mathrm{~nm}$ of silicon dioxide $\left(\mathrm{SiO}_{2}\right)$ by RF-bias and setting the following 
parameters: $600 \mathrm{~W}$ power, $0.2 \mathrm{~Pa}$ pressure, $5 \mathrm{sccm}$ argon gas flow and deposition time of $42 \mathrm{~s}$. $\mathrm{SiO}_{2}$ promotes surface wettability and adhesion of the succeeding metasurface coating, also increasing its homogeneity and reducing the overall nanoroughness. On top of it, we ran deposition cycles with alternating titania $\left(\mathrm{TiO}_{2}\right)$ and gold $(\mathrm{Au})$ targets, up to a total number of 8 layers. For each Au deposition burst, we operated the tool for $3 \mathrm{~s}$ at $50 \mathrm{~W}$ DC-bias and 0.6 Pa pressure, while each deposition of $\mathrm{TiO}_{2}$ took $43 \mathrm{~s}$, using a $600 \mathrm{~W}$ RF field at $0.6 \mathrm{~Pa}$ pressure. The metasurface was terminated with an extra $\mathrm{TiO}_{2}$ layer, deposited for $72 \mathrm{~s}$. Finally, the metasurfaces were coated with a hydrophobic pPFDA layer in the same way as previously described in the case of silicon. Out of the pool of samples with different geometrical characteristics, we selected the ones with the following dimensions: $d \approx 2.5 \mu \mathrm{m}, s \approx 5.0 \mu \mathrm{m}$ and $h \approx 3.3 \mu \mathrm{m}$. The wetting fraction, $\varphi=\pi d^{2} / 4 s^{2}$ , was $\sim 20 \%$.

b. Control surfaces. We fabricated the superhydrophobic control surfaces in almost the same manner as the plasmonic composite surfaces, with the only difference of omitting sputter deposition, meaning that curing of the PUA micropillars was directly followed by iCVD deposition of a hydrophobic pPFDA coating.

c. Sample for metasurface coating cross-section depiction. To demonstrate the multilayer nature of our metasurface coating, we deposited the metasurface similar to the aforementioned procedure on a smooth silicon substrate, omitting the $\mathrm{SiO}_{2}$ layer, since no dewetting issues were observed in this case. We kept all deposition parameters the same and adjusted the total number of layers to 44 .

d. Hierarchical plasmonic composite surfaces. We fabricated our hierarchical surfaces in two sets of steps. For the first roughness tier (micropillars) we followed the procedure described in $a$. For the second tier (nanoroughness), we prepared a $\sim 2$ wt. $\%$ of Capstone ${ }^{\circledR}$ ST-100HS polymer dispersion in ethanol, in which we added a $\sim 1.3$ wt.\% dispersion of HFS nanoparticles, which we 
then sonicated for 3 min using a probe sonicator. The mean specific surface area of the HFS powder is $260 \mathrm{~m}^{2} \mathrm{~g}^{-1}$. We regulated the $\mathrm{pH}$ of the total dispersion to values of $\sim 4$ to ensure good dispersibility of both the polymer and HFS. Finally, we sprayed the dispersion onto our micropillars from a distance of 15-20 cm using a Paasche airbrush at an air pressure of 3 bar. Solvent evaporation was ensured by drying the surfaces on a hot plate set at $50^{\circ} \mathrm{C}$ for $>10 \mathrm{~min}$.

\section{Characterization}

We characterized the topography of the control, plasmonic composite and hierarchical surfaces by means of stylus profilometry (Bruker Dektak XT) and scanning electron microscopy (SEM; Hitachi SU8230). For the SEM micrographs of micropillars (with or without the nanorough coating), we set the acceleration voltage at $1-2 \mathrm{kV}$ and utilized the secondary electron and lower detectors to collect surface and topographic information. For the cross-sectional micrograph, we selected the aforementioned detectors and raised the voltage to $5 \mathrm{kV}$. We measured all apparent static, advancing and receding contact angles with an OCA 35 goniometer (DataPhysics), with the tilting method and for droplet volumes of $10 \mu \mathrm{L}$. These angles were, in the case of the hierarchical plasmonic composite, $\theta^{*}=159^{\circ} \pm 3^{\circ}, \theta_{\mathrm{a}}^{*}=167^{\circ} \pm 1^{\circ}$ and $\theta_{\mathrm{r}}^{*}=150^{\circ} \pm 3^{\circ}$, respectively. We carried out transparency, reflectivity and absorption measurements on our surfaces with the help of a V700 (Jasco) UV-VIS spectrometer with an integrating sphere.

\section{Experimental setup and protocols}

We conducted surface temperature calibrations using either a high-speed infrared camera (SC7500, FLIR), for surfaces illuminated with halogen light (Flexilux 600 longlife), with $P=1$ $\mathrm{kW} \mathrm{m}{ }^{-2}$ and $P=2 \mathrm{~kW} \mathrm{~m}^{-2}$, or a resistance temperature detector in the case of sunlike illumination with a xenon arc source (66902, Newport), equipped with an AM1.5 air mass filter to render it a solar simulator, for $P=1 \mathrm{~kW} \mathrm{~m}^{-2}$ and $3.5 \mathrm{~kW} \mathrm{~m}^{-2}$. The diameter of the light spot was $\sim 8 \mathrm{~mm}$ for 
halogen light and $>2 \mathrm{~cm}$ for the solar simulator. With the latter, we were able to homogeneously illuminate the whole sample. We assumed thermal equilibrium at $t=300 \mathrm{~s}$ (light was switched on at $t=0 \mathrm{~s})$ and each measurement was run three times. Light from the solar simulator was stabilized by waiting for at least $30 \mathrm{~min}$ prior to running any experiments. (See Figure $1 \mathrm{f}$ for a schematic of the experimental configuration during calibration and Figure $1 \mathrm{~g}$ for the temperature increase caused by the solar simulator on control and plasmonic composite surfaces.) We performed the warm droplet experiments when the illuminated surfaces reached thermal equilibrium and waited $\sim 1$ min between subsequent droplet dispensations. (See Supporting Information, section "Experimental setup, water temperature calibration and droplet cooling rate" and Figure S7, for more details on the experimental setups used for calibrating the water temperature and running the warm droplet experiments.) The droplet or impact position on a sample was shifted after every experiment to ensure minimal systematic effects of surface defects on our experiments and statistics. The relative humidity of the room was in the $40 \%-55 \%$ range throughout the study.

\section{Supporting Information}

The Supporting Information is available online free of charge. The following sections are included:

Topography of the control; transparency, reflectivity and absorption of superhydrophobic control surfaces; mechanical durability of the metasurface coating; inhibiting fog with sunlight in plasmonic composite surfaces; enhanced impalement resistance with concentrated light; theoretical basis of impalement criterion; effect of surface temperature on condensation nucleation on superhydrophobic control surfaces; experimental setup, water temperature calibration and droplet cooling rate; effect of light on pressure-driven impalement in plasmonic composite surfaces; comparison of droplet retraction dynamics; mapping droplet impact behavior on plasmonic composite 
surfaces; effusion in a nanopore; heterogeneous condensation nucleation; light-driven repellency of dynamic warm droplets (video).

\section{Author contributions statement}

T.M.S. and D.P. designed the research and provided scientific advice throughout its progression; E.M., H.L. and S.T. performed experiments and analyzed data; E.M. and H.L. fabricated and characterized the surfaces; E.M., H.L., T.M.S. and D.P. wrote the paper.

\section{Acknowledgements}

Partial support of the Swiss National Science Foundation under grant number 162565 and the European Research Council under Advanced Grant 669908 (INTICE) are acknowledged. We thank Ute Drechsler (IBM Rüschlikon) for assistance in surface fabrication. We also thank Hyunchul Park for advice on UV-curable polymers and fabrication protocols, and Karl Schlichting (LTNT, ETH Zurich) for insights into effusion theory.

\section{Additional information}

Competing financial interests: The authors declare no competing financial interests. 


\section{References}

(1) Barthlott, W.; Neinhuis, C. Purity of the Sacred Lotus, or Escape from Contamination in Biological Surfaces. Planta 1997, 202, 1-8.

(2) Zheng, Y.; Gao, X.; Jiang, L. Directional Adhesion of Superhydrophobic Butterfly Wings. Soft Matter 2007, 3, 178-182.

(3) Gao, X.; Jiang, L. Water-Repellent Legs of Water Striders. Nature 2004, 432, 36.

(4) Blossey, R. Self-Cleaning Surfaces-Virtual Realities. Nat. Mater. 2003, 2, 301-306.

(5) Genzer, J.; Efimenko, K. Recent Developments in Superhydrophobic Surfaces and Their Relevance to Marine Fouling: A Review. Biofouling 2006, 22, 339-360.

(6) Jung, S.; Tiwari, M. K.; Doan, N. V.; Poulikakos, D. Mechanism of Supercooled Droplet Freezing on Surfaces. Nat. Commun. 2012, 3, 615 .

(7) Mishchenko, L.; Hatton, B.; Bahadur, V.; Taylor, J. A.; Krupenkin, T.; Aizenberg, J. Design of Ice-Free Nanostructured Surfaces Based on Repulsion of Impacting Water Droplets. ACS Nano 2010, 4, 7699-7707.

(8) Lai, Y.; Gao, X.; Zhuang, H.; Huang, J.; Lin, C.; Jiang, L. Designing Superhydrophobic Porous Nanostructures with Tunable Water Adhesion. Adv. Mater. 2009, 21, 3799-3803.

(9) Maitra, T.; Jung, S.; Giger, M. E.; Kandrical, V.; Ruesch, T.; Poulikakos, D. Superhydrophobicity vs Ice Adhesion: The Quandary of Robust Icephobic Surface Design. Adv. Mater. Interfaces 2015, 2, 1500330.

(10) Boreyko, J. B.; Collier, C. P. Dewetting Transitions on Superhydrophobic Surfaces: When Are Wenzel Drops Reversible? J. Phys. Chem. C 2013, 117, 18084-18090.

(11) Feng, L.; Li, S.; Li, Y.; Li, H.; Zhang, L.; Zhai, J.; Song, Y.; Liu, B.; Jiang, L.; Zhu, D. Super-Hydrophobic Surfaces: From Natural to Artificial. Adv. Mater. 2002, 14, 1857-1860.

(12) Liu, M.; Wang, S.; Jiang, L. Nature-Inspired Superwettability Systems. Nat. Rev. Mater. 
2017, 2, 17036.

(13) McCarthy, M.; Gerasopoulos, K.; Enright, R.; Culver, J. N.; Ghodssi, R.; Wang, E. N. Biotemplated Hierarchical Surfaces and the Role of Dual Length Scales on the Repellency of Impacting Droplets. Appl. Phys. Lett. 2012, 100, 263701.

(14) Patankar, N. A. Mimicking the Lotus Effect: Influence of Double Roughness Structures and Slender Pillars. Langmuir 2004, 20, 8209-8213.

(15) Soto, D.; Ugur, A.; Farnham, T. A.; Gleason, K. K.; Varanasi, K. K. Short-Fluorinated ICVD Coatings for Nonwetting Fabrics. Adv. Funct. Mater. 2018, 28, 1707355.

(16) Peng, C.; Chen, Z.; Tiwari, M. K. All-Organic Superhydrophobic Coatings with Mechanochemical Robustness and Liquid Impalement Resistance. Nat. Mater. 2018, 17, $355-360$.

(17) Tuteja, A.; Choi, W.; Ma, M.; Mabry, J. M.; Mazzella, S. A.; Rutledge, G. C.; McKinley, G. H.; Cohen, R. E. Designing Superoleophobic Surfaces. Science 2007, 318, 1618-1622.

(18) Wu, S. Polymer Interface and Adhesion; Taylor \& Francis: Boca Raton, 1982.

(19) Vasileiou, T.; Gerber, J.; Prautzsch, J.; Schutzius, T. M.; Poulikakos, D. Superhydrophobicity Enhancement through Substrate Flexibility. Proc. Natl. Acad. Sci. 2016, 113, 13307-13312.

(20) Vasileiou, T.; Schutzius, T. M.; Poulikakos, D. Imparting Icephobicity with Substrate Flexibility. Langmuir 2017, 33, 6708-6718.

(21) Cassie, A. B. D.; Baxter, S. Wettability of Porous Surfaces. Trans. Faraday Soc. 1944, 40, $546-551$.

(22) Wenzel, R. N. Resistance of Solid Surfaces to Wetting by Water. Ind. Eng. Chem. 1936, 28, 988-994.

(23) Maitra, T.; Tiwari, M. K.; Antonini, C.; Schoch, P.; Jung, S.; Eberle, P.; Poulikakos, D. On 
the Nanoengineering of Superhydrophobic and Impalement Resistant Surface Textures below the Freezing Temperature. Nano Lett. 2014, 14, 172-182.

(24) Reyssat, M.; Pépin, A.; Marty, F.; Chen, Y.; Quéré, D. Bouncing Transitions on Microtextured Materials. Europhys. Lett. 2006, 74, 306-312.

(25) Moulinet, S.; Bartolo, D. Life and Death of a Fakir Droplet: Impalement Transitions on Superhydrophobic Surfaces. Eur. Phys. J. E 2007, 24, 251-260.

(26) Mouterde, T.; Lecointre, P.; Lehoucq, G.; Checco, A.; Clanet, C.; Quéré, D. Two Recipes for Repelling Hot Water. Nat. Commun. 2019, 10, 1410.

(27) Bartolo, D.; Bouamrirene, F.; Verneuil, É.; Buguin, A.; Silberzan, P.; Moulinet, S. Bouncing or Sticky Droplets: Impalement Transitions on Superhydrophobic Micropatterned Surfaces. Europhys. Lett. 2006, 74, 299-305.

(28) Cheng, Y. T.; Rodak, D. E. Is the Lotus Leaf Superhydrophobic? Appl. Phys. Lett. 2005, 86, 144101.

(29) Liu, Y.; Chen, X.; Xin, J. H. Can Superhydrophobic Surfaces Repel Hot Water? J. Mater. Chem. 2009, 19, 5602-5611.

(30) Wilke, K. L.; Preston, D. J.; Lu, Z.; Wang, E. N. Toward Condensation-Resistant Omniphobic Surfaces. ACS Nano 2018, 12, 11013-11021.

(31) Maitra, T.; Antonini, C.; Tiwari, M. K.; Mularczyk, A.; Imeri, Z.; Schoch, P.; Poulikakos, D. Supercooled Water Drops Impacting Superhydrophobic Textures. Langmuir 2014, 30, $10855-10861$.

(32) Schutzius, T. M.; Jung, S.; Maitra, T.; Eberle, P.; Antonini, C.; Stamatopoulos, C.; Poulikakos, D. Physics of Icing and Rational Design of Surfaces with Extraordinary Icephobicity. Langmuir 2015, 31, 4807-4821.

(33) Cao, L.; Jones, A. K.; Sikka, V. K.; Wu, J.; Gao, D. Anti-Icing Superhydrophobic Coatings. 
Langmuir 2009, 25, 12444-12448.

(34) Boreyko, J. B.; Chen, C. H. Self-Propelled Jumping Drops on Superhydrophobic Surfaces. Phys. Fluids 2010, 22, 091110.

(35) Miljkovic, N.; Enright, R.; Nam, Y.; Lopez, K.; Dou, N.; Sack, J.; Wang, E. N. JumpingDroplet-Enhanced Condensation on Scalable Superhydrophobic Nanostructured Surfaces. Nano Lett. 2013, 13, 179-187.

(36) Zhu, P.; Chen, R.; Wang, L. Topography-Directed Hot-Water Super-Repellent Surfaces. Adv. Sci. 2019, 1900798.

(37) Wan, F.; Yang, D.-Q.; Sacher, E. Repelling Hot Water from Superhydrophobic Surfaces Based on Carbon Nanotubes. J. Mater. Chem. A 2015, 3, 16953-16960.

(38) Walker, C.; Lerch, S.; Reininger, M.; Eghlidi, H.; Milionis, A.; Schutzius, T. M.; Poulikakos, D. Desublimation Frosting on Nanoengineered Surfaces. ACS Nano 2018, 12, 8288-8296.

(39) Nakajima, A.; Hashimoto, K.; Watanabe, T.; Takai, K.; Yamauchi, G.; Fujishima, A. Transparent Superhydrophobic Thin Films with Self-Cleaning Properties. Langmuir 2000, $16,7044-7047$.

(40) Li, J.; Wan, H.; Ye, Y.; Zhou, H.; Chen, J. One-Step Process to Fabrication of Transparent Superhydrophobic SiO2 Paper. Appl. Surf. Sci. 2012, 261, 470-472.

(41) Yu, S.; Guo, Z.; Liu, W. Biomimetic Transparent and Superhydrophobic Coatings: From Nature and beyond Nature. Chem. Commun. 2015, 51, 1775-1794.

(42) Neumann, O.; Urban, A. S.; Day, J.; Lal, S.; Nordlander, P.; Halas, N. J. Solar Vapor Generation Enabled by Nanoparticles. ACS Nano 2013, 7, 42-49.

(43) Neumann, O.; Feronti, C.; Neumann, A. D.; Dong, A.; Schell, K.; Lu, B.; Kim, E.; Quinn, M.; Thompson, S.; Grady, N.; Nordlander, P.; Oden, M.; Halas, N. J. Compact Solar 
Autoclave Based on Steam Generation Using Broadband Light-Harvesting Nanoparticles. Proc. Natl. Acad. Sci. 2013, 110, 11677-11681.

(44) Ni, G.; Li, G.; Boriskina, S. V.; Li, H.; Yang, W.; Zhang, T.; Chen, G. Steam Generation under One Sun Enabled by a Floating Structure with Thermal Concentration. Nat. Energy 2016, $1,16126$.

(45) Brongersma, M. L.; Halas, N. J.; Nordlander, P. Plasmon-Induced Hot Carrier Science and Technology. Nat. Nanotechnol. 2015, 10, 25-34.

(46) Naldoni, A.; Shalaev, V. M.; Brongersma, M. L. Applying Plasmonics to a Sustainable Future. Science 2017, 356, 908-909.

(47) Adleman, J. R.; Boyd, D. A.; Goodwin, D. G.; Psaltis, D. Heterogenous Catalysis Mediated by Plasmon Heating. Nano Lett. 2009, 9, 4417-4423.

(48) Zhou, L.; Tan, Y.; Wang, J.; Xu, W.; Yuan, Y.; Cai, W.; Zhu, S.; Zhu, J. 3D Self-Assembly of Aluminium Nanoparticles for Plasmon-Enhanced Solar Desalination. Nat. Photonics 2016, 10, 393-398.

(49) Ni, G.; Zandavi, S. H.; Javid, S. M.; Boriskina, S. V.; Cooper, T. A.; Chen, G. A SaltRejecting Floating Solar Still for Low-Cost Desalination. Energy Environ. Sci. 2018, 11, $1510-1519$.

(50) Kuzyk, A.; Schreiber, R.; Zhang, H.; Govorov, A. O.; Liedl, T.; Liu, N. Reconfigurable 3D Plasmonic Metamolecules. Nat. Mater. 2014, 13, 862-866.

(51) Mitridis, E.; Schutzius, T. M.; Sicher, A.; Hail, C. U.; Eghlidi, H.; Poulikakos, D. Metasurfaces Leveraging Solar Energy for Icephobicity. ACS Nano 2018, 12, 7009-7017.

(52) Walker, C.; Mitridis, E.; Kreiner, T.; Eghlidi, H.; Schutzius, T. M.; Poulikakos, D. Transparent Metasurfaces Counteracting Fogging by Harnessing Sunlight. Nano Lett. 2019, $19,1595-1604$. 
(53) Almutawa, F.; Vandal, R.; Wang, S. Q.; Lim, H. W. Current Status of Photoprotection by Window Glass, Automobile Glass, Window Films, and Sunglasses. Photodermatol. Photoimmunol. Photomed. 2013, 29, 65-72.

(54) Ji, L.; Kim, J.-K.; Ji, Q.; Leung, K.-N.; Chen, Y.; Gough, R. A. Conformal Metal Thin-Film Coatings in High-Aspect-Ratio Trenches Using a Self-Sputtered Rf-Driven Plasma Source. J. Vac. Sci. Technol. B Microelectron. Nanom. Struct. 2007, 25, 1227-1230.

(55) Jennings, S. . The Mean Free Path in Air. J. Aerosol Sci. 1988, 19, 159-166.

(56) Hanel, D. Molekulare Gasdynamik: Einführung in die kinetische Theorie der Gase und Lattice-Boltzmann-Methoden; Springer-Verlag: Berlin, 2004.

(57) Atkins, P.; De Paula, J. Atkin's Physical Chemistry, 8th Ed; Oxford University Press: New York, 2006.

(58) Eberle, P.; Tiwari, M. K.; Maitra, T.; Poulikakos, D. Rational Nanostructuring of Surfaces for Extraordinary Icephobicity. Nanoscale 2014, 6, 4874-4881.

(59) Gupta, M.; Gleason, K. K. Initiated Chemical Vapor Deposition of Poly $(1 \mathrm{H}, 1 \mathrm{H}, 2 \mathrm{H}, 2 \mathrm{H}-$ Perfluorodecyl Acrylate) Thin Films. Langmuir 2006, 22, 10047-10052. 


\section{Figures}

$\mathbf{a}$
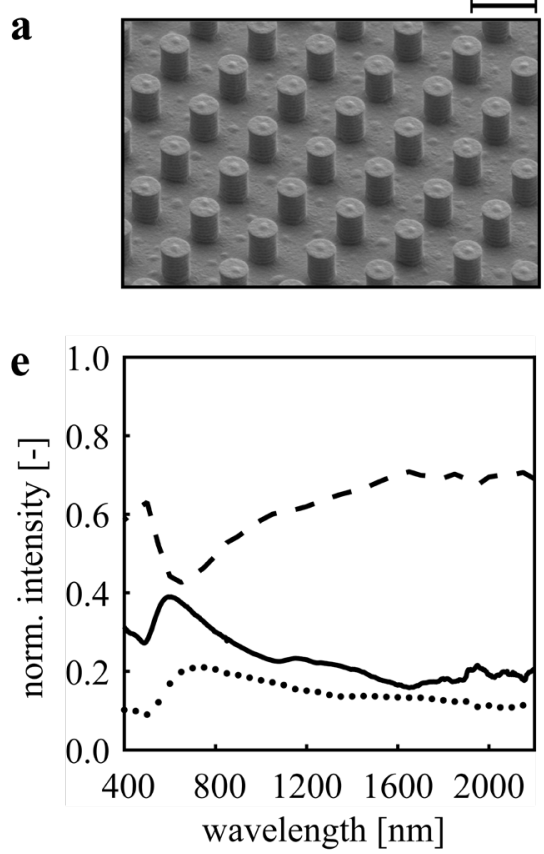
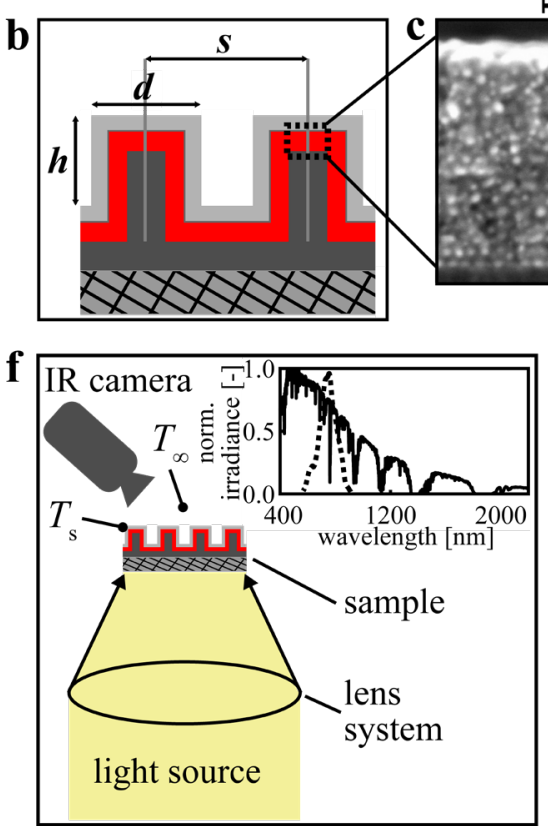
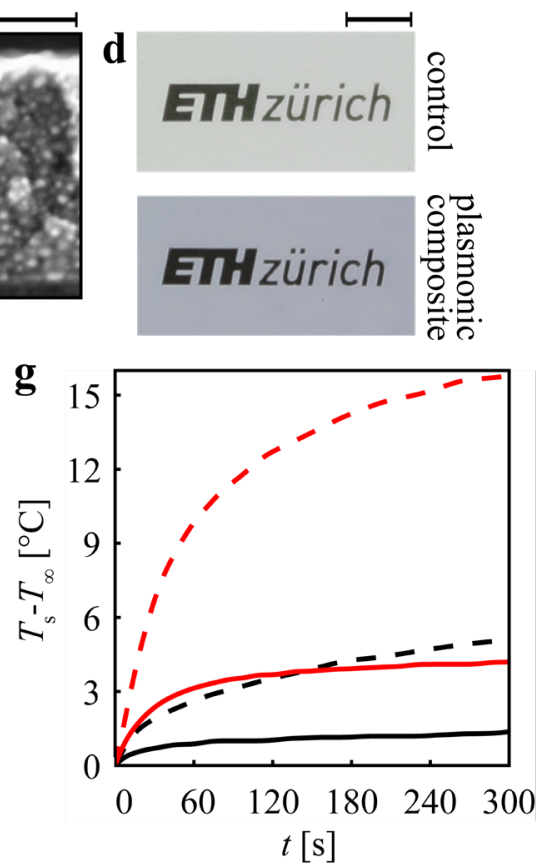

Figure 1. Superhydrophobic plasmonic composite surface. (a) Micrograph showing the surface from a tilted-view perspective. (b) Schematic of the surface cross-section, revealing its different layers. It consists of a hydrophobic fluoropolymer layer (top, light gray); plasmonic metasurface coating (red); micropillar array (dark gray); and glass substrate (gray, black hatch). (c) Micrograph showing the cross-section of a representative metasurface coating; the thickness here is exaggerated to facilitate imaging (the metasurface coating used throughout this study had a thickness of $\sim 60 \mathrm{~nm})$. The pillar diameter $(d)$, height $(h)$, and spacing $(s)$ are shown in $\mathbf{b}$. The sample in a has $[d, s, h]=[2.5,5.0,3.3] \mu \mathrm{m}$. (d) The high transparency of the plasmonic composite surface in comparison to a control surface (lacks the metasurface coating) is evident in the readability of a printed logo placed directly underneath it. (e) Transparency $(---)$, reflectivity $(\cdot \cdots)$, and absorption (- ) spectra of the surface shown in a. (f) Schematic of the experimental setup used to measure the temperature change of the samples relative to ambient upon illumination. The normalized irradiance spectra of the light sources we used in this study are shown as inset plots (solar simulator, ; halogen lamp, - - ). (g) Surface temperature change relative to ambient, $T_{\mathrm{s}}-T_{\infty}, v s$ time, $t$, of control $\left(P=1 \mathrm{~kW} \mathrm{~m}^{-2}\right.$, black curve; $P=3.5 \mathrm{~kW} \mathrm{~m}^{-2}$, black dashed curve $)$ and plasmonic composite $\left(P=1 \mathrm{~kW} \mathrm{~m}{ }^{-2}\right.$, red curve; $P=3.5 \mathrm{~kW} \mathrm{~m}^{-2}$, red dashed curve) surfaces. The samples are illuminated when $t>0 \mathrm{~s}$ with a solar simulator. Each curve represents the mean values of $n=3$ experiments. The logos in $\mathbf{d}$ were reprinted with permission from ETH Zurich. Copyright 2020 ETH Zurich. Scale bars: (a), $5 \mu \mathrm{m}$; (c), $100 \mathrm{~nm}$; (d), $5 \mathrm{~mm}$. 


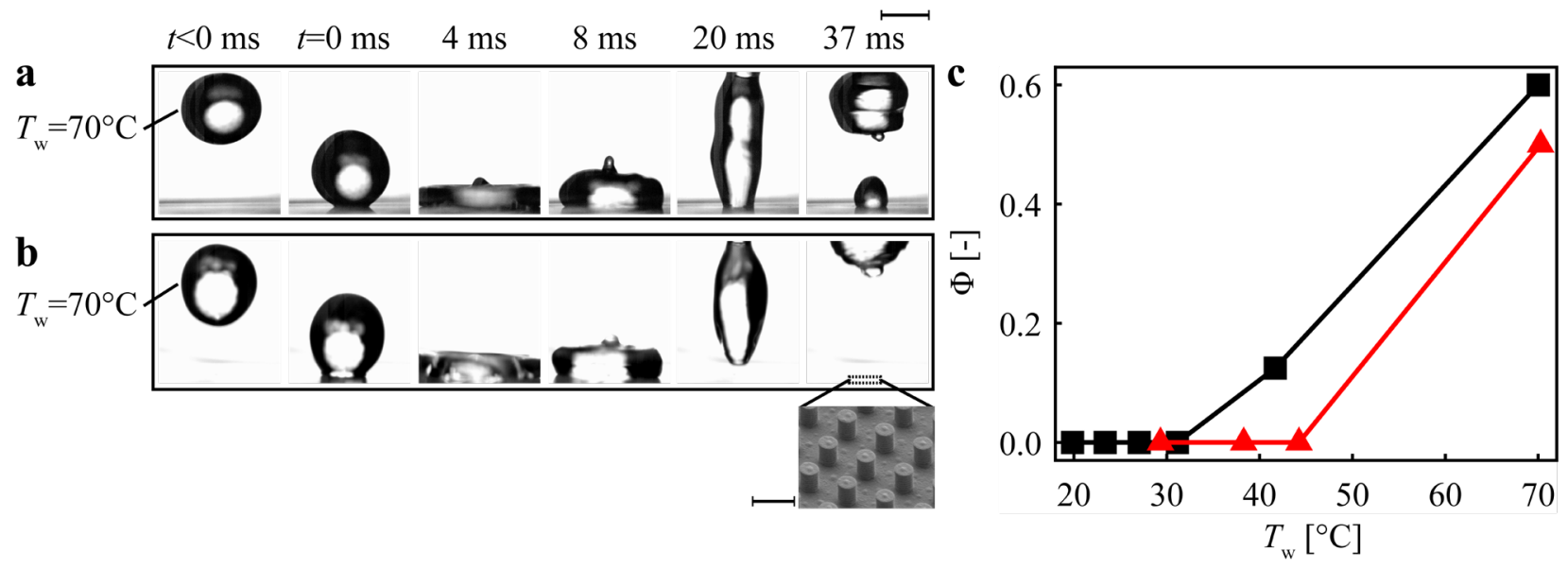

Figure 2. Enhancing superhydrophobicity with one sun. Image sequences showing warm droplets impacting onto a superhydrophobic plasmonic composite surface (a) without and (b) under illumination (solar simulator; $P=1 \mathrm{~kW} \mathrm{~m}^{-2}$ ). For all experiments here, $W e=26$ and $T_{\infty}=21^{\circ} \mathrm{C}$. A micrograph of the surface is shown as an inset image. (c) Probability of droplet impalement, $\Phi, v_{S}$ water droplet temperature, $T_{\mathrm{w}}$, for no illumination (black squares) and under illumination (red triangles) conditions. Each data point represents $n \geq 7$ experiments. Scale bars: (a)-(b), 2 mm; inset in (b), $5 \mu \mathrm{m}$. 

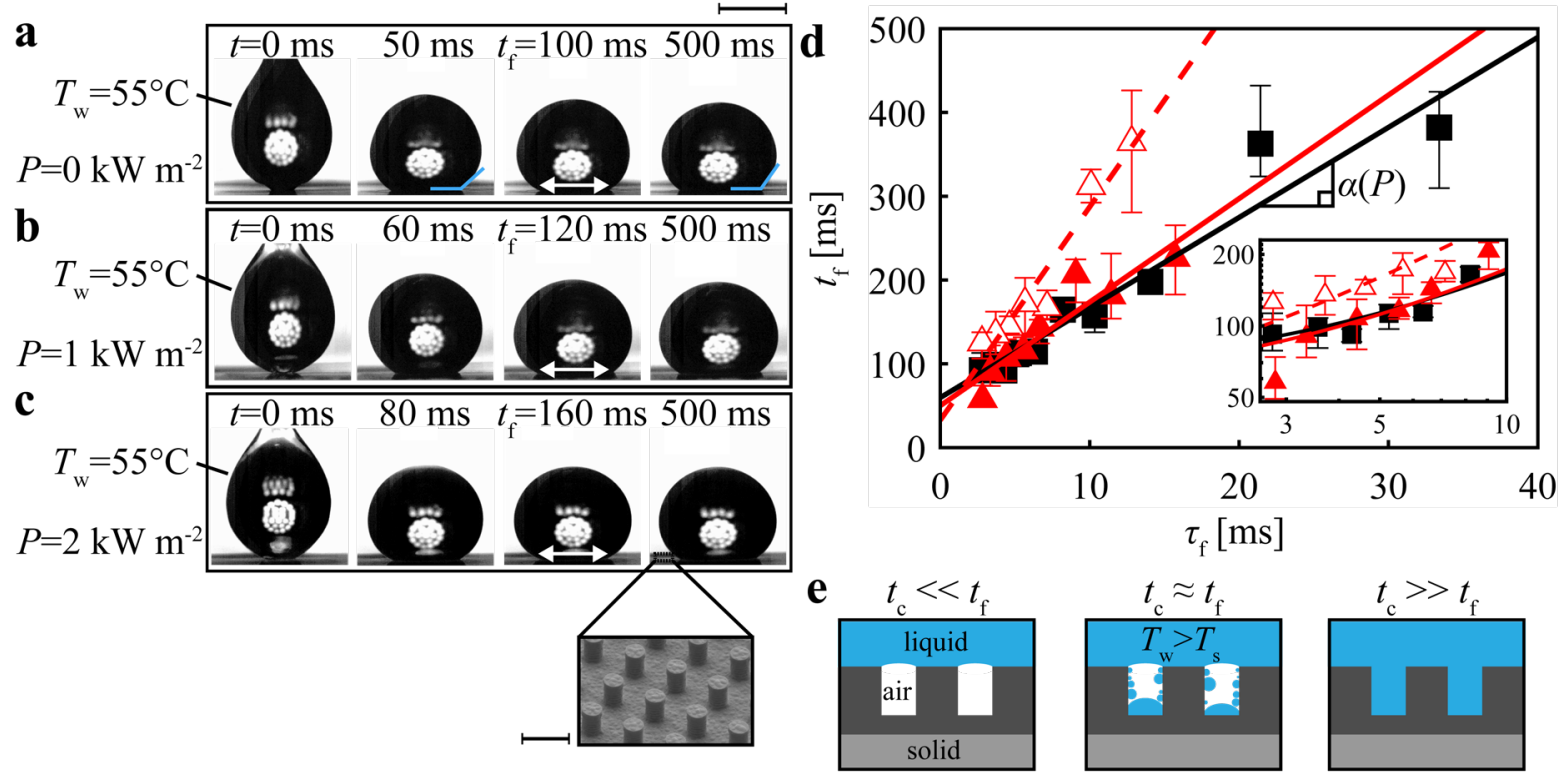

Figure 3. Interplay between light coupling, plasmonic composite, and warm droplet repellency. (a)-(c) Image sequences showing the wetting behavior of warm water droplets on a superhydrophobic plasmonic composite surface for $\mathbf{a}, P=0 \mathrm{~kW} \mathrm{~m}^{-2}, \mathbf{b}, P=1 \mathrm{~kW} \mathrm{~m}^{-2}$, and $\mathbf{c}, P=2 \mathrm{~kW}$ $\mathrm{m}^{-2}\left(T_{\infty}=22^{\circ} \mathrm{C}\right)$. We used a halogen lamp to illuminate the samples. (d) Droplet impalement time, $t_{\mathrm{f}}, v s$ theoretical cavity filling time, $\tau_{\mathrm{f}}$, for $P=0 \mathrm{~kW} \mathrm{~m}^{-2}$ (black squares and line), $P=1 \mathrm{~kW} \mathrm{~m}^{-2}$ (red filled triangles and red line), and $P=2 \mathrm{~kW} \mathrm{~m}^{-2}$ (red open triangles and dashed red line). Each data point represents $n=3$ experiments, and the lower and upper error values represent the minimum and maximum measured values, respectively. The three lines of best fit have slopes of $\alpha \approx 11$ (for confidence $C=95 \%, \alpha=[8,13]), 12(C=95 \%, \alpha=[8,17])$, and $26(C=95 \%, \alpha=[18,33])$ corresponding to $P=0,1$, and $2 \mathrm{~kW} \mathrm{~m}^{-2}$, respectively. The magnified inset, plotted in logarithmic axes, clarifies the behavior of the warmest droplets. (e) Schematic showing the mechanism of condensation impalement that occurs when a warm droplet is placed on the surface. Scale bars: (a)-(c), 2mm; inset in (c), $5 \mu \mathrm{m}$. 


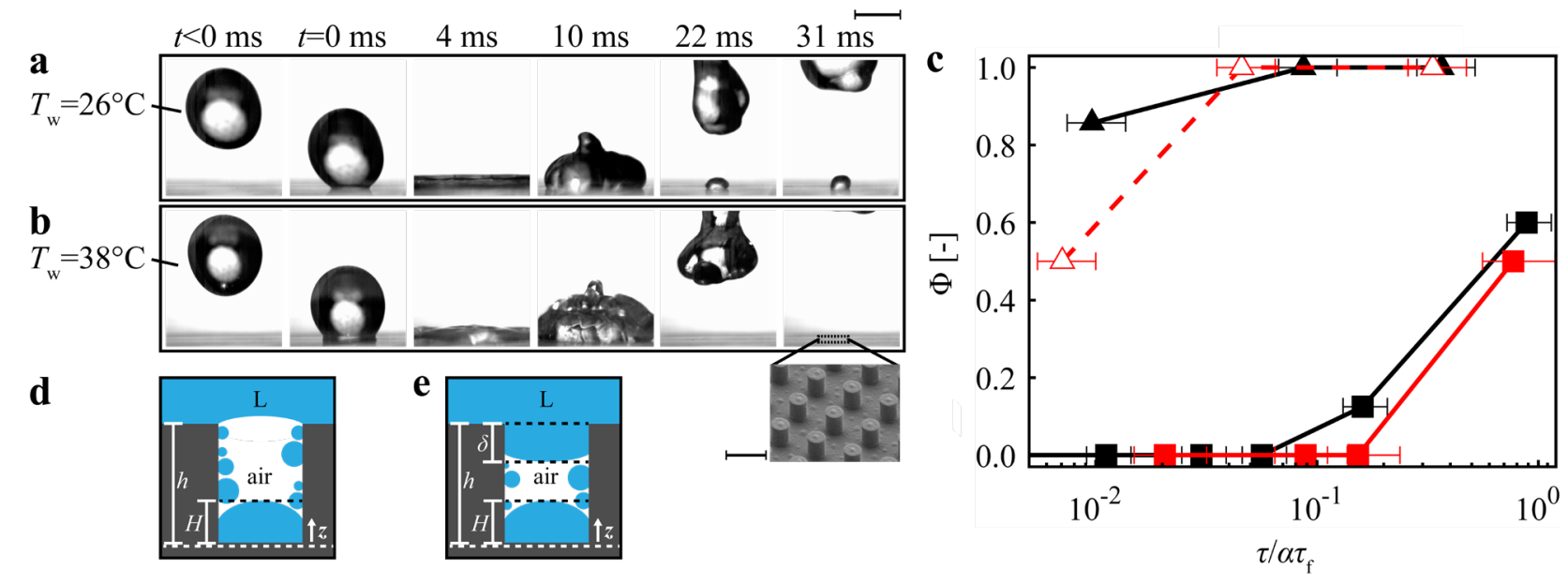

Figure 4. Mechanisms of light-enhanced superhydrophobicity. (a)-(b) Image sequences of warm water droplets impacting onto a superhydrophobic plasmonic composite surface $\left(T_{\infty}=21^{\circ} \mathrm{C}\right)$ for $\mathbf{a}, W e=73, P=0 \mathrm{~kW} \mathrm{~m}^{-2}$, and $\mathbf{b}, W e=73, P=3.5 \mathrm{~kW} \mathrm{~m}^{-2}$. A solar simulator was used for illumination. (c) Impalement probability, $\Phi$, vs ratio of droplet oscillation time to condensate filling time, $\tau / \alpha \tau_{\mathrm{f}}$, for $W e=26$ and $P=0 \mathrm{~kW} \mathrm{~m}^{-2}$ (black squares); $W e=26$ and $P=1 \mathrm{~kW} \mathrm{~m}^{-2}$ (red filled squares and red line); $W e=73$ and $P=0 \mathrm{~kW} \mathrm{~m}^{-2}$ (black triangles); and $W e=73$ and $P=3.5 \mathrm{~kW}$ $\mathrm{m}^{-2}$ (red open triangles and dashed red line). The error bars are based on the $95 \%$ confidence level in the values of $\alpha$, which we previously calculated. Each data point represents $n \geq 7$ experiments. (d)-(e) Schematics of impalement mechanisms; d, condensation-driven, and e, condensation and pressure-driven (combined). Scale bars: (a)-(b), $2 \mathrm{~mm}$; inset in (b), $5 \mu \mathrm{m}$. 

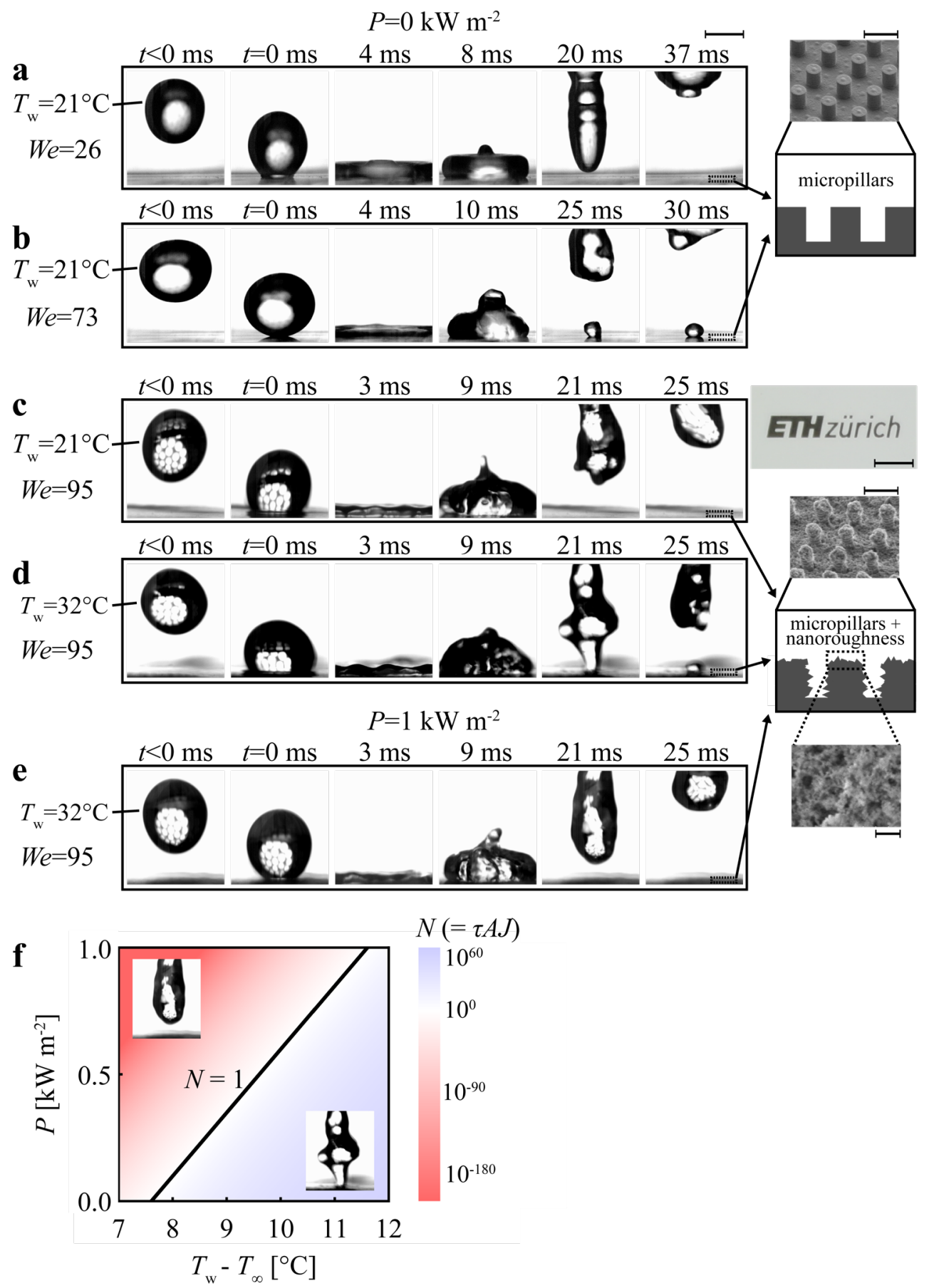

Figure 5. Repellency boost with added nanoroughness and sunlight. (a)-(b) Image sequences showing ambient water droplets $\left(T_{\infty}=21^{\circ} \mathrm{C}\right)$ impacting onto our control surface for $\mathbf{a}, W e=26$, and $\mathbf{b}, W e=73$. (c)-(d) Image sequences of water droplets impacting onto a hierarchical plasmonic composite for $\mathbf{c}, W e=95, T_{\mathrm{w}}=T_{\infty}=21^{\circ} \mathrm{C}$, and $\mathbf{d}, W e=95, T_{\mathrm{w}}=32^{\circ} \mathrm{C}$. In all cases, a-d, the surfaces were not illuminated $\left(P=0 \mathrm{~kW} \mathrm{~m}^{-2}\right)$. (e) Image sequence of a warm water droplet $\left(T_{\mathrm{w}}=32^{\circ} \mathrm{C}\right)$ impacting onto the hierarchical surface for $W e=95$. Here the surface was illuminated $(P=1 \mathrm{~kW}$ $\mathrm{m}^{-2}$ ). The inset in $\mathbf{c}$ shows a picture of the hierarchical plasmonic composite with a printed logo placed underneath it. Two insets in a-e show schematic representations and a scanning electron micrograph for each roughness tier. (f) Plot of incident solar power density, $P$, vs $T_{\mathrm{w}}-T_{\infty}, v s$ the 
number of water nuclei per nanopore, $N(=\tau A J)$, during droplet impact on the hierarchical plasmonic composite, for a fixed $T_{\mathrm{w}}=32^{\circ} \mathrm{C}$ and a range of $T_{\infty}<T_{\mathrm{w}} . N=1$ is considered as probable for condensation nucleation. Cylindrical pores of depth and diameter of $50 \mathrm{~nm}$ were assumed. The logo in c, inset, was reprinted with permission from ETH Zurich. Copyright 2020 ETH Zurich. Scale bars: (a)-(e), $2 \mathrm{~mm}$; inset in (a)-(b), $5 \mu \mathrm{m}$; inset picture in (c), $5 \mathrm{~mm}$; inset in (c)-(e), $5 \mu \mathrm{m}$ (micropillars), $200 \mathrm{~nm}$ (nanoroughness). 


\section{TOC}

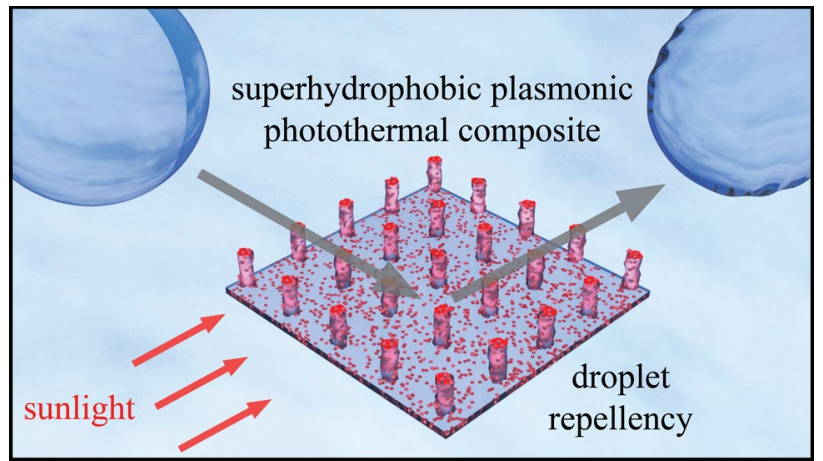

Warm water repellency with sunlight and superhydrophobic plasmonic photothermal composite surfaces. 